\title{
ON THE INDEPENDENCE OF PRINCIPAL MINORS OF DETERMINANTS*
}

BY

\author{
E. B. STOUFFER
}

\section{INTRODUCTION}

A principal minor, or coaxial minor, of a determinant $A$ is a minor obtained by striking out from $A$ the same rows as columns. There are $2^{n}-1$ principal minors of a determinant of the $n$th order, the determinant itself being included, but only $n^{2}-n+1$ of them are independent. $t$

For $n=1,2,3$ the principal minors are all independent and for $n=4$ the relations between them have been quite extensively studied of For $n>4$ little has been published either as to which minors constitute an independent set or as to the relations between the minors.

It is one of the purposes of this paper to determine several different types of complete sets of independent principal minors of the general determinant of the $n$th order and to show how the elements of the determinant may be expressed in terms of the minors of an independent set.

If we have a second determinant of the $n$th order with elements independent of the elements of the first determinant, there is a definite set of determinants obtained by replacing one or more columns of the first determinant by the corresponding column or columns of the second determinant. The set of principal minors of all such determinants is greatly enlarged over the set from the single determinant. It is the second purpose of this paper to determine several types of complete sets of independent principal minors of this enlarged set. There is also determined in this paper a complete set of independent principal minors of the determinants obtained when the above process is extended by adjoining to the original determinant more than one determinant of the $n$th order with independent elements.

- Presented to the Society, April 14, 1922, and December 1, 1923.

† See MacMahon, Philosophical Transactions of the Royal Society of L ond on, vol. 185, pp. 111-160; Muir, Phil 'sophical Magazine and Journal of S cience, ser. 5, vol. 38, pp. 537-541.

† See, e.g., MacMahon, loc. cit.; Nansen, Philos ophical Magazine and Journal of Science, ser. 5, vol. 44, pp. 362-67; Muir, Transactions of the Royal Society of E d in b u rg h, vol. 39, pp. 323-339. 
It is the third purpose of this paper to prove the independence of certain sums of principal minors. In the fourth section of the paper the independence of these sums is used to determine the possibility of expressing polynomials as determinants with linear elements.

Let $A$ represent a determinant of the $n$th order in which the element in row $i$ and column $j$ is denoted by $a_{i j}$. Furthermore, let $\left(A_{k l m n} \ldots\right)$ denote the principal minor obtained by striking out in $A$ all columns and rows except those numbered $k, l, m, n, \cdots$. Thus, ${ }^{*}$

$$
\left(A_{i}\right)=a_{i i}, \quad\left(A_{i j}\right)=\left|\begin{array}{ll}
a_{i i} & a_{i j} \\
a_{j i} & a_{j j}
\end{array}\right|, \cdots
$$

It has been shown by Muirt that the values of the principal minors of a determinant $A$ with general elements $a_{i j}$ are not affected if certain sets of $n-1$ of the elements are put equal to unity. For instance, if we divide the elements of the second column by $a_{12}$, of the third column by $a_{13}$, of the fourth column by $a_{14}$, and so forth, and also multiply the elements of the second row by $a_{12}$, of the third row by $a_{13}$, of the fourth row by $a_{14}$, and so forth, there is no effect either upon the values of the principal minors or upon the independence of the individual elements, except that $a_{1 j}=1(j=2,3, \cdots, n)$. The fact that there are only $n^{2}-n+1$ independent elements remaining is Muir's proof that there cannot be more than $n^{2}-n+1$ independent principal minors.

It may be shown similarly that there is no loss of generality in assuming $a_{i j}=1(i+1=j)$ in place of $a_{1 j}=1$.

TheOREM I. The minors

$$
\left(A_{i}\right), \quad\left(A_{i j}\right), \quad\left(A_{1 i j}\right) \quad(i, j=1,2, \ldots, n ; i<j)
$$

constitute a total of $n^{2}-n+1$ independent principal minors.

We shall prove this theorem by showing that the functional matrix of these minors with respect to the elements $a_{i j}$ contains a non-vanishing determinant of order $n^{2}-n+1$. In order to simplify the process we shall assume that $a_{1 j}=1(j=2,3, \cdots, n)$ and also that, after the partial derivatives with respect to the remaining $a_{i j}$ have been formed, the elements $a_{i j}(i>j>1)$ are all put equal to zero. Then all $\partial\left(A_{i}\right) / \partial a_{m l}=0$ except

\footnotetext{
*We shall assume throughout this paper that the subscripts in the symbols for principal minors have been arranged in order of ascending magnitude.

† Muir, Philosophical Magazine and Journal of Science, ser.5, vol. 38, pp. 537-541.
} 
that $\partial\left(A_{i}\right) / \partial a_{i i}=1$; all $\partial\left(A_{i j}\right) / \partial a_{m l}=0(1<m, m \neq l)$ except that $\partial\left(A_{i j}\right) / \partial a_{j i}=-a_{i j} ;$ all $\partial\left(A_{1 i j}\right) / \partial a_{m l}=0(1<m<l)$ except that $\partial\left(A_{1 i j}\right) / \partial a_{i j}=a_{j 1}$. Under these conditions there is in the functional matrix a determinant of order $n^{2}-n+1$ which may be expressed as the product of the three determinants

$$
\frac{\partial\left[\left(A_{1}\right),\left(A_{2}\right), \cdots,\left(A_{n}\right)\right]}{\partial\left[a_{11}, a_{22}, \cdots, a_{n n}\right]}
$$

(3) $\frac{\partial\left[\left(A_{12}\right),\left(A_{18}\right), \cdots,\left(A_{1 n}\right),\left(A_{23}\right),\left(A_{24}\right), \cdots,\left(A_{2 n}\right), \cdots,\left(A_{n-1, n}\right)\right]}{\partial\left[a_{21}, a_{31}, \cdots, a_{n 1}, a_{32}, a_{42}, \cdots, a_{n 2}, \cdots, a_{n, n-1}\right]}$,

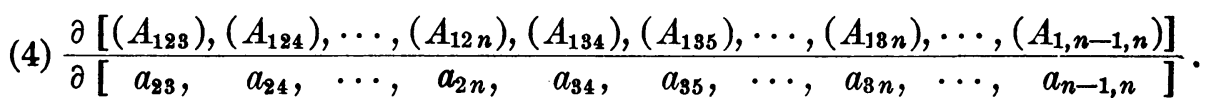

These determinants are all different from zero since in each of them the elements of the principal diagonal are different from zero and all other elements are equal to zero. The minors (1) are therefore independent.

The explicit expressions for $A$ and for the principal minors of $A$ in terms of the minors (1) are obtained by expressing the elements of $A$ in terms of the minors (1). Under the condition that $a_{1 j}=1(j>1)$, we have

$$
\begin{aligned}
\left(A_{i}\right) & =a_{i i}, \\
\left(A_{1 j}\right) & =a_{11} a_{j j}-a_{j 1}, \\
\left(A_{i j}\right) & =a_{i i} a_{j j}-a_{i j} a_{j i} \quad(i>1), \\
\left(A_{1 i j}\right) & =a_{11} A_{i j}-a_{i 1} a_{j j}+a_{j 1} a_{i j}+a_{i 1} a_{j i}-a_{j 1} a_{i i},
\end{aligned}
$$

whence

$$
\begin{aligned}
a_{i i} & =\left(A_{i}\right) \\
a_{j 1} & =\left(A_{1}\right)\left(A_{j}\right)--\left(A_{1 j}\right), \\
a_{i j} a_{j i} & =\left(A_{i}\right)\left(A_{j}\right)-\left(A_{i j}\right) \quad(i>1), \\
a_{j 1} a_{i j}+a_{i 1} a_{j i} & =\left(A_{1 i j}\right)-\left(A_{1}\right)\left(A_{i j}\right)+2\left(A_{1}\right)\left(A_{i}\right)\left(A_{j}\right)-\left(A_{j}\right)\left(A_{1 i}\right)-\left(A_{i}\right)\left(A_{1 j}\right) .
\end{aligned}
$$

The expressions for all the elements of $A$ in terms of $\left(A_{i}\right),\left(A_{i j}\right),\left(A_{1 i j}\right)$ follow immediately from these equations.*

Another complete set of independent principal minors of $A$ is obtained if the minors $\left(A_{1 i j}\right)$ of the set $(1)$ are replaced by the minors $\left(A_{12 j}\right),\left(A_{122 j}\right)$,

* MacMahon, (Philosophical Transactions of the Royal Society of London, vol. 185, p. 147) states that it is impossible to express a determinant of odd order in terms of its principal minors. By the above process this statement is shown to be incorrect. 
$\left(A_{1234 j}\right), \cdots,\left(A_{128} \cdots n\right)$, where $j$ in each case takes all the values up to $n$ which are greater than the subscript immediately preceding it. The functional matrix will again be used in proving the independence of this set of $n^{2}-n+1$ principal minors. If we assume as before that $a_{1 j}=1(j=2,3, \cdots, n)$ and that $a_{i j}(i>j>1)$ are put equal to zero after the partial derivatives are formed, the matrix contains a determinant of order $n^{2}-n+1$ which consists of the product of the determinants (2) and (3) and the determinant

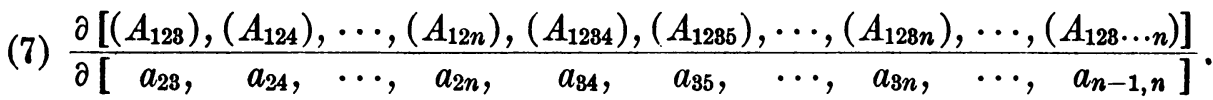

Under the above conditions

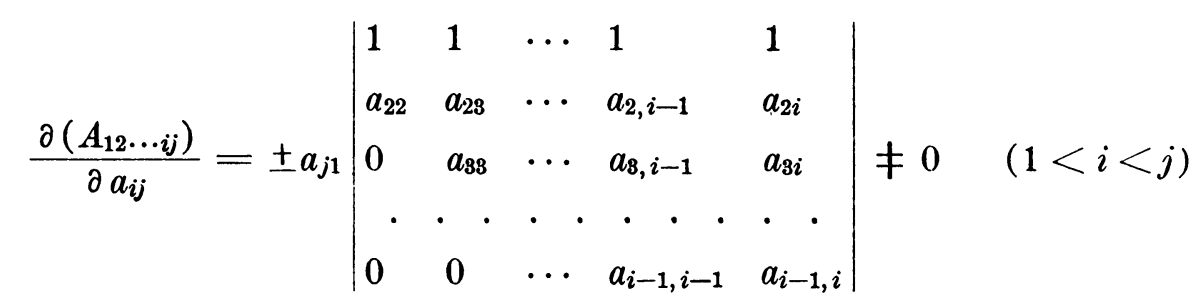

and

$$
\frac{\partial\left(A_{12} \ldots i j\right)}{\partial a_{m l}}=0 \quad(m<l ; m>i \text { or } l>j) .
$$

Consequently, in the determinant (7) all elements above the principal diagonal are equal to zero and all elements of the principal diagonal are different from zero. We thus have

Theorem II. The minors

$$
\left(A_{i}\right), \quad\left(A_{i j}\right), \quad\left(A_{12 j}\right), \quad\left(A_{123 j}\right), \quad \cdots, \quad\left(A_{123 \cdots n}\right)
$$

form a complete set of independent principal minors of the general determinant $A$.

It is easily verified that the process of obtaining the expressions for the elements of $A$ in terms of this second set of principal minors involves merely the successive solution of sets of two equations, one of which is linear and the other quadratic. However, the pairs of equations to be solved are not so easily written as before, since each pair has a different form from those which precede it.

Still another set of independent principal minors is obtained if the $\frac{1}{2}(n-1)(n-2)$ minors $\left(A_{1 i j}\right)$ of the set (1) are replaced by the minors $\left(A_{123}\right),\left(A_{234}\right),\left(A_{345}\right), \cdots,\left(A_{1234}\right),\left(A_{2345}\right), \cdots,\left(A_{128} \cdots n\right)$. In order to 
prove them independent, let us assume that $a_{i j}=1(i+1=j)$, instead of $a_{1 j}=1$, and that $a_{i j}(i>j+1)$ are put equal to zero after the partial derivatives in the functional matrix are formed. Under these conditions the matrix contains a determinant of order $n^{2}-n+1$ which consists of the product of two non-vanishing determinants of the same form as (2) and (3) and the determinant

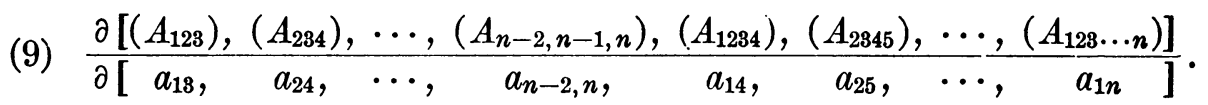

The latter determinant obviously has all elements of the principal diagonal different from zero and all elements above the principal diagonal equal to zero. We thus have

Theorem III. The minors

$$
\left(A_{i}\right), \quad\left(A_{i j}\right), \quad\left(A_{i, i+1, i+2, \cdots, i+r}\right) \quad(r=2,3, \cdots, n-1 ; i+r \leqq n)
$$

form a complete set of independent principal minors of the general determinant $A$.

In order to express the elements of $A$ in terms of the minors (10) it is again necessary to solve rather complicated pairs of equations, each pair consisting of a linear and a quadratic equation.

II

Let $B$ denote a second determinant of the $n$th order with elements $b_{i j}$. A series of determinants of the $n$th order may be obtained by replacing one or more columns of $A$ by the corresponding column or columns of $B$. Each of these new determinants gives a set of $2^{n}-1$ principal minors.

Let us indicate by $\left(A_{i j k \ldots} \ldots B_{r s t} \ldots\right)$ the principal minor which belongs to the above set and which contains elements from columns $i, j, k, \ldots(i<j<k \ldots)$ of the determinant $A$ and from columns $r, s, t, \ldots(r<s<t \ldots)$ of the determinant $B$. It is evident that the numbers $i, j, k, \cdots, r, s, t, \cdots$ must all be different.

Just as in the previous case, we can make $n-1$ of the elements, say $a_{1 j}(j=2,3, \cdots, n)$ or $a_{i j}(i+1=j)$, equal to unity without affecting the values of the principal minors. It follows that there are not more than $2 n^{2}-n+1$ independent principal minors.

Theorem IV. The minors

$$
\left(A_{i}\right),\left(B_{i}\right),\left(A_{1 i}\right),\left(A_{1} B_{i}\right),\left(B_{1 i}\right),\left(A_{1 i j}\right),\left(A_{1 i} B_{j}\right),\left(A_{1} B_{i j}\right),\left(B_{1 i j}\right)
$$


form a complete set of independent principal minors of the determinants obtained by combining $A$ and $B$.

In order to prove the set (11) independent let us first observe that an element $a_{l m}, b_{l m}(l \neq m)$ can occur only in minors having a subscript equal to $l$ and another subscript equal to $m$. It follows that the functional matrix of the set (11) contains a determinant of order $2 n^{2}-n+1$ con. sisting of the product of the determinants

$$
\frac{\partial\left[\left(A_{1}\right),\left(A_{2}\right), \cdots,\left(A_{n}\right)\right]}{\partial\left[a_{11}, a_{22}, \cdots, a_{n n}\right]}, \quad \frac{\partial\left[\left(B_{1}\right),\left(B_{2}\right), \cdots,\left(B_{n}\right)\right]}{\partial\left[\begin{array}{ll}
b_{11}, & b_{22}, \cdots, b_{n n}
\end{array}\right]}
$$

and of determinants of the forms

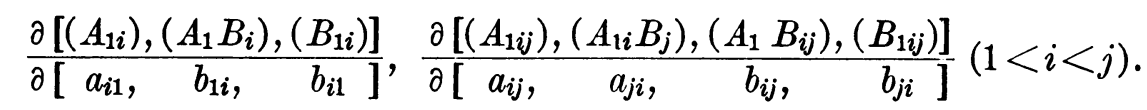

The determinants (12) are already known to be different from zero. If, after forming the determinants (13), we put $a_{i j}=0(i>j>1)$ and $b_{i j}=0(i>j)$, it is immediately evident that each of them is equal to the product of the elements in its principal diagonal, all of which are different from zero.

By analogous processes we may prove

THEOREM V. The set of principal minors

$$
\left(A_{j}\right), \quad\left(B_{j}\right), \quad\left(A_{1 j}\right), \quad\left(A_{1} B_{j}\right), \quad\left(B_{1 j}\right),
$$

$\left(A_{1,2,8, \cdots, i-1, i, j}\right),\left(A_{1,2,8, \cdots, i-1, i} B_{j}\right),\left(A_{1,2,3, \cdots, i-1} B_{i j}\right),\left(A_{2,3, \cdots, i-1} B_{1 i j}\right)$

and the set

$$
(i \geqq 2) \text {, }
$$

$$
\begin{gathered}
\left(A_{j}\right), \quad\left(B_{j}\right), \quad\left(A_{j, j+1}\right), \quad\left(A_{j} B_{j+1}\right), \quad\left(B_{j, j+1}\right), \quad\left(A_{i, i+1, \cdots, i+r}\right), \\
\left(A_{i, i+1, \cdots, i+r-1} B_{i+r}\right),\left(A_{i, i+1, \cdots, i+r-2} B_{i+r-1, i+r}\right),\left(A_{i+1, \cdots, i+r-2} B_{i, i+r-1, i+r}\right) \\
(r=2,3, \cdots, n-1 ; i+r \leqq n)
\end{gathered}
$$

each form a complete set of independent principal minors of the determinants obtained by combining $A$ and $B$.

It should be noted that the sets (11), (14) and (15) are obtained from the sets (1), (8) and (10), respectively, by adjoining to each set the minors obtained by replacing in the determinants of the set the elements of the last column, the elements of the last two columns, and the elements of the first column and the last two columns, by the corresponding elements 
$b_{i j}$, except that only a part of the minors of the second order thus obtained are used.

The $n^{2}$ minors

$$
\left(B_{i}\right), \quad\left(A_{i} B_{j}\right), \quad\left(B_{i j}\right) \quad(i, j=1,2, \cdots, n ; i<j)
$$

when arranged in the above order each contain an element $b_{i j}$ not in the minors which precede it and not in the minors of $A$. We thus have

THEOREM VI. The minors (16) together with any complete and independent set of principal minors of $A$ form a complete set of independent principal minors of the determinants formed by combining $A$ and $B$.

The process of expressing the elements $a_{i j}$ and $b_{i j}$ in terms of the minors of any one of the above independent sets involves the simultaneous solution of linear and quadratic equations. The process can be carried out quite easily for the set composed of the minors (16) and $\left(A_{i}\right),\left(A_{i j}\right),\left(A_{1 i j}\right)$.

The above process of forming determinants by combining two determinants $A$ and $B$ may be extended by combining $k$ determinants $A, B$, $C, D, \ldots$ of the $n$th order. Select for the first column of the new determinant the first column of one of the $k$ determinants, for the second column the second column of one of the $k$ determinants, and so forth. The principal minors of all such determinants of the $n$th order will now be considered for the purpose of determining a complete and independent set.

The symbol $\left(A_{i j k} \ldots B_{r s t} \ldots C_{u v w} \ldots\right)$ will be used to indicate the principal minor containing elements from columns $i, j, k, \cdots$ of $A$, from columns $r, s, t, \cdots$ of $B$, from columns $u, v, w, \cdots$ of $C$, and so forth.

Since $n-1$ of the $k n^{2}$ elements may again be assumed equal to unity, there cannot be more than $k n^{2}-n+1$ independent principal minors obtained from the whole set of combined determinants.

Among the minors of the set (11) there is a group in which elements of $B$ appear in the last column only and another group in which elements of $B$ appear in the last two columns only. If in each of the minors of the first group the elements of the last column are replaced by the corresponding elements of $C, D, \cdots$, successively, and if in each of the minors of the second group the elements of the next to the last column are replaced by the corresponding elements of $C, D, \cdots$, successively, we have, for each determinant $C, D, \cdots, n^{2}$ new principal minors. That each minor thus found is independent of the others and of the minors of the set (11) is evident from the fact that, with the proper arrangement, each of them contains an element $c_{i j}, d_{i j}, \ldots$ not in any of the preceding minors. Moreover, since the number of independent principal minors thus obtained is $k n^{2}-n+1$, the set is complete. 
By exactly the same process we can obtain complete sets of minors for the enlarged system from either (14) or (16). The set (15) yields a complete set for $A, B, C, D, \ldots$ if the elements of the last column of the minors which contain a single column of elements from $B$ and the elements of the first column of the minors which contain two columns of elements from $B$ are replaced by the corresponding elements of $C, D, \cdots$, successively.

The minors to be added to any one of the sets (11), (14), (15), (16) may take many other forms, but the above systems are especially simple because the elements of $C, D, \ldots$ appear only linearly. The fact that all elements of $A, B, C, D, \ldots$ can be expressed in terms of the principal minors follows immediately from the fact that the elements of $C, D, \ldots$ appear linearly and from the fact that we have been able previously to express the elements of $A$ and $B$ in terms of principal minors.

\section{III}

Let us denote by $I_{\alpha}$ the sum of all the principal minors of order $\alpha$ of the determinant $A$ of order $n$. That the $n$ sums thus obtained are independent becomes evident if we put $a_{i j}=0(i \neq j)$ and observe that the sums $I_{\alpha}(\alpha=1,2, \ldots, n)$ are then simply the $n$ elementary symmetric functions of $a_{i i}(i=1,2, \cdots, n)$.

In the previous section we obtained new determinants of the $n$th order by combining the determinants $A, B, C, D, \ldots$. We shall now study the sums $I_{\alpha \beta \gamma \ldots}$ of the principal minors of these new determinants, where $I_{\alpha \beta \gamma \ldots}$ denotes the sum of all such principal minors containing $\alpha$ columns from $A, \beta$ columns from $B, \gamma$ columns from $C$, and so forth.

We shall first find a superior limit to the number of independent sums $I_{\alpha \beta \gamma} \ldots$ obtained by the combination of two or more determinants. For this purpose let us observe that $I_{\alpha \beta \gamma} \ldots$ may be obtained from $A$ by successive applications of two different types of differential operators. The first type is of the form

$$
D_{a} \equiv \sum_{i=1}^{n} \frac{\partial}{\partial a_{i i}} .
$$

If the $k$ th successive application is denoted by $k ! D_{a}^{k}$, we have

$$
D_{a} A=I_{n-1}, \quad D_{a}^{2} A=I_{n-2}, \quad \cdots, \quad D_{a}^{k} A=I_{n-k} .
$$

The second type is of the form

$$
D_{a b} \equiv \sum_{i=1}^{n} \sum_{j=1}^{n} b_{i j} \frac{\partial}{\partial a_{i j}} .
$$


If the $l$ th successive application is denoted by $l ! D_{a b}^{l}$, we have

$D_{a b} I_{n-k}=I_{n-k-1,1}, \quad D_{a b}^{2} I_{n-k}=I_{n-k-2,2}, \quad \cdots, \quad D_{a b}^{\ell} I_{n-k}=I_{n-k-l, l}$.

The operator

$$
D_{b c}=\sum_{i=1}^{n} \sum_{j=1}^{n} c_{i j} \frac{\partial}{\partial b_{i j}},
$$

which is of the second type, applied successively gives

$$
D_{b c} I_{n-k-l, l}=I_{n-k-l, l-1,1}, \quad \ldots, \quad D_{b c}^{m} I_{n-k-l, l}=I_{n-k-l, l-m, m} .
$$

Thus the sum $I_{\alpha \beta \gamma \ldots}$ may be obtained from $A$ by first operating with $D_{a}$ successively $n-(\alpha+\beta+\gamma+\cdots)$ times, by then operating with $D_{a b}$ successively $\beta+\gamma+\ldots$ times, by then operating with $D_{b c}$ successively $\gamma+\cdots$ times, and so forth.

We shall next show that all $I_{\alpha \beta \gamma} \ldots$ are solutions of the system of differential equations

$$
\begin{gathered}
R_{r s}(f) \equiv \sum_{k=1}^{n}\left(a_{k r} \frac{\partial f}{\partial a_{k s}}-a_{s k} \frac{\partial f}{\partial a_{r k}}+b_{k r} \frac{\partial f}{\partial b_{k s}}-b_{s k} \frac{\partial f}{\partial b_{r k}}+\cdots\right)=0 \\
(r, s=1,2, \cdots, n) .
\end{gathered}
$$

The determinant $I_{n}=A$ is evidently a solution of every equation of (17). It is easy to see that the relation

$$
R_{r s}\left(D_{a} A\right)-D_{a}\left(R_{r s} A\right)=-\left(\frac{\partial A}{\partial a_{r s}}-\frac{\partial A}{\partial a_{r s}}\right)=0
$$

is true, since terms containing the second derivatives vanish. But $R_{r s}(A)=0$, whence it follows that $R_{r s}\left(D_{a} A\right)=R_{r s}\left(I_{n-1}\right)=0$. Therefore, $I_{n-1}$ is a solution of the system (17) and by induction it follows at once that all $I_{\alpha}$ are solutions of (17).

Let us repeat the above process with the operator $D_{a b}$ instead of $D_{a}$. We have

$$
\begin{aligned}
R_{r s}\left(D_{a b} I_{\alpha}\right)-D_{a b}\left(R_{r s} I_{\alpha}\right) & =\sum_{k=1}^{n}\left[\left(b_{k r} \frac{\partial I_{\alpha}}{\partial a_{k s}}-b_{s k} \frac{\partial I_{\alpha}}{\partial a_{r k}}\right)\right. \\
& \left.-\left(b_{k r} \frac{\partial I_{\alpha}}{\partial a_{k s}}-b_{s k} \frac{\partial I_{\alpha}}{\partial a_{r k}}\right)\right]=0 .
\end{aligned}
$$

Since $R_{r s}\left(I_{\alpha}\right)=0$, we have $R_{r s}\left(D_{a b} I_{\alpha}\right)=0$. It follows that $I_{\alpha 1}$ is a solution of (17) and by induction it is again easily shown that all $I_{\alpha \beta}$ are solutions. 
A continuation of the above process shows that all $I_{\alpha \beta \gamma \ldots}$ are solutions of (17).

The one relation $\sum_{r=1}^{n} R_{r r}(f)=0$ between the equations (17) is obvious. That the remaining $n^{2}-1$ equations are independent for two or more sets of elements $a_{i j}, b_{i j}, c_{i j}, \cdots$ is easily seen by writing out the matrix of the equation and putting $a_{i k}=0(i \neq k)$ in this matrix.

The system (17) thus involves $k n^{2}$ variables, where $k$ is the number of the determinants $A, B, C, \cdots$, and contains $n^{2}-1$ independent equations for $k>1$. It follows that for $k>1$ there cannot be more than $k n^{2}-\left(n^{2}-1\right)$ independent sums $I_{\alpha \beta \gamma \ldots} \ldots$

It is interesting to observe that for $k=2$ and $n>2$ the number $\frac{1}{2}\left(n^{2}+3 n\right)$ of sums $I_{\alpha \beta}$ is less than the superior limit $n^{2}+1$ fixed by the system of differential equations. As a matter of fact, there are $\frac{1}{2}\left(n^{2}-3 n+2\right)$ other independent solutions of equations (17) in this case.*

We shall now prove

Theorem VII. The sums

$$
I_{\alpha \beta \gamma} \quad(\alpha, \beta=0,1,2, \cdots, n ; \gamma=0,1,2,3)
$$

form a complete and independent system for $k=3$.

It is evident that there are $n$ sums of the type $I_{\alpha}$ and $\frac{1}{2} n(n+1)$ of the type $I_{\alpha \beta}(\beta=1,2, \cdots, n)$. Consequently, there are $\frac{1}{2} n(n+1)$ of the type $I_{\alpha \beta 1}, \frac{1}{2} n(n+1)-n$ of the type $I_{\alpha \beta 2}, \frac{1}{2} n(n+1)-(2 n-1)$ of the type $I_{\alpha \beta 3}$. These minors make a total of $2 n^{2}+1$.

Let us now put $a_{i j}=0(i \neq j)$ and investigate the resulting functional matrix of the sums (18) with respect to $a_{i i}, b_{i j}, c_{i j}$. There exists in this matrix a determinant of maximum order which consists of the product of the determinants

(19) $\frac{\partial\left[I_{1}, I_{2}, \cdots, I_{n}\right]}{\partial\left[a_{11}, a_{22}, \cdots, a_{n n}\right]}, \frac{\partial\left[I_{01}, I_{11}, \cdots, I_{n-1,1}\right]}{\partial\left[b_{11}, b_{22}, \cdots, b_{n n}\right]}, \frac{\partial\left[I_{001}, I_{101}, \cdots, I_{n-1,0,1}\right]}{\partial\left[c_{11}, c_{22}, \cdots, c_{n n}\right]}$,

and of a determinant $F$ consisting of the functional determinant of all the remaining sums of (18) with respect to all the elements $b_{i j}, c_{i j}(i \neq j)$ except $b_{i, i+1}(i=1,2, \cdots, n-1)$. The three determinants (19) are different from zero since each is equal to the functional determinant of the $n$ symmetric functions of $a_{11}, a_{22}, \cdots, a_{n n}$.

We shall prove that $F$ is different from zero by showing that the coefficient of the highest power of $a_{n n}$ which occurs in $F$ is different from

*Cf. Stouffer, Proceedings of the London Mathematical Society, ser. 2, vol. 15, p. 222. 
zero. This coefficient is the product of the determinant which corresponds to $F$ when the order of $A$ is $n-1$ and the functional determinant

(20) $\frac{\partial\left[I_{02}, I_{011}, I_{002}, I_{03}, I_{021}, I_{012}, I_{008}, \cdots, I_{0 n}, I_{0, n-1,1}, I_{0, n-2,2}, I_{0, n-8,8}\right]}{\partial\left[b_{n, n-1}, c_{n, n-1}, c_{n-1, n}, b_{n, n-2}, c_{n, n-2}, b_{n-2, n}, c_{n-2, n}, \cdots, b_{n 1}, c_{n 1}, b_{1 n}, c_{1 n}\right]}$.

After forming the partial derivatives in (20) let us put all $b_{i k}(i \neq k)$ and $c_{i k}(i \neq k)$ equal to zero except $b_{i, i+1}(i=1,2, \cdots, n-1)$, $b_{i+1, i}(i=1,2, \cdots, n-3)$, and $c_{i+1, i}(i=1,2, \cdots, n-1)$. A partial derivative of $I_{0 \beta \gamma}$ with respect to $b_{n r}, b_{r n}, c_{n r}$ or $c_{r n}$ is then zero unless at least one of the determinants in $I_{0 \beta \gamma}$ contains elements from the $r$ th row and column and also from all rows and columns of higher number. It follows that (20) becomes the product of the determinants

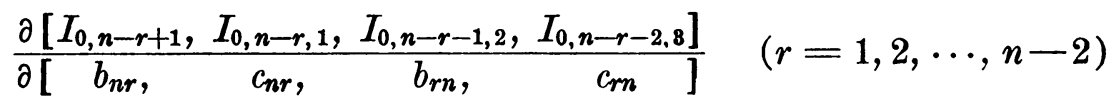

and the determinant

$$
\frac{\partial\left[I_{02}, I_{011}, I_{002}\right]}{\partial\left[b_{n, n-1}, c_{n, n-1}, c_{n-1, n}\right]} .
$$

Each of the determinants (21) and (22) is different from zero since, under the above conditions upon $b_{i j}$ and $c_{i j}$, each is the product of the elements of its principal diagonal, all of which are different from zero. Thus $F$ does not vanish when the order of $A$ is $n$, provided it does not vanish when the order of $A$ is $n-1$. But it is easily verified that $F$ is different from zero for $n=3$. Hence, by induction the sums (18) are proved to be independent. Since the set (18) contains the maximum number of independent sums, it is a complete system.

The nature of the determinants (19) and the fact that (20) reduces to the product of the elements of its principal diagonal prove the independence of the sums (18) and

$$
I_{\alpha \beta 0 \delta \varepsilon \ldots,}, \quad I_{\alpha 01 \delta \varepsilon \ldots}, \quad I_{\alpha \beta 2 \delta \varepsilon \ldots} \quad(\delta+\varepsilon+\cdots=1),
$$

for $k=4,5, \cdots$, and any $n$, provided that they are independent for $n=3$. This latter fact is easily verified.

Since the set (23) contains $n^{2}$ sums for $\delta=1, n^{2}$ sums for $\varepsilon=1$, and so forth, we have proved

TheOREM VIII. The sums

(24) $I_{\alpha \beta \gamma}, I_{\alpha \beta 0 \delta \varepsilon \ldots}, I_{\alpha 01 \delta \varepsilon \ldots}, I_{\alpha \beta 2 \delta \varepsilon \ldots} \quad(\gamma=0,1,2,3 ; \delta+\varepsilon+\ldots=1)$

form a complete and independent system for any number $k$ of combined determinants of any order $n$. 
IV

Dickson* has determined the types of general homogeneous polynomials which are expressible as determinants with linear elements. We shall now show that his main results follow very easily from our knowledge of the independence of sums of principal minors of determinants.

Let $f\left(x_{1}, x_{2}, \cdots, x_{k}\right)$ represent a general homogeneous polynomial of degree $n$ in $k$ variables. We assume that the coefficient of the $n$th power of one of the variables, say $x_{1}$, is unity. There is no loss of generality here since the coefficient can always be made unity by a linear transformation. If it is possible to express $f$ as a determinant with linear elements, a combination of rows and columns will always bring that determinant to the form

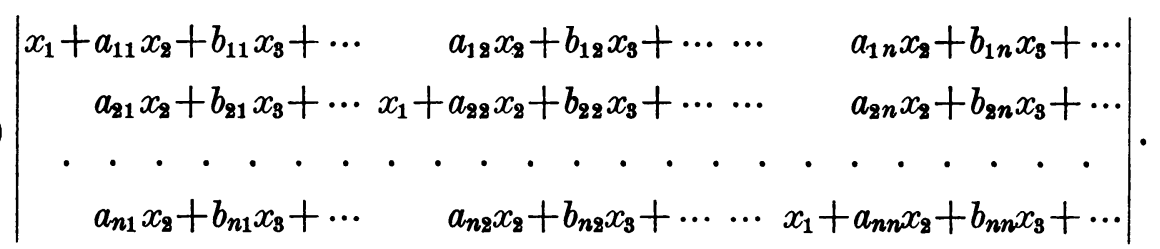

The expansion of (25) gives a polynomial in which each coefficient after the first is one of the sums $I_{\alpha \beta \gamma \ldots}$ and, conversely, each sum $I_{\alpha \beta \gamma} \ldots(\alpha, \beta, \gamma, \ldots=0,1,2, \cdots, n)$ appears as a coefficient, the number of subscripts in $I_{\alpha \beta \gamma} \ldots$ being $k-1$. Since this polynomial must be exactly $f$, we have a proof of

Theorem IX. A general polynomial of degree $n$ in $k$ variables cannot be expressed as a determinant with linear elements if it has in addition to $x_{1}^{n}$ more than $(k-2) n^{2}+1$ terms if $k>3$ or more than $\frac{1}{2}\left(n^{2}+3 n\right)$ terms if $k=3$.

This is Dickson's first theorem except that he included the case $k=3$ in the general theorem. For $k=3$ the limit is $\frac{1}{2}\left(n^{2}+3 n\right)$ which for $n>2$ is smaller than $n^{2}+1$, the limit for $k=3$ in the general theorem.

Theorem X. Any determinant of order $n$ whose elements are linear homogeneous functions of $x_{1}, x_{2}, \cdots, x_{k}$ can be expressed in a canonical form involving not more than $(k-2) n^{2}+2$ parameters for $k>3$ and not more than $\frac{1}{2}\left(n^{2}+3 n\right)+1$ parameters for $k=3$.

This is Dickson's generalization of his first theorem except for the case $k=3$. Its proof follows immediately from the maximum number of independent sums which appear as the coefficients in the expansion of the determinant. The additional parameter arises from the fact that the

* These Transactions, vol. 22 (1921), pp. 167-179. 
coefficient of $x_{1}^{n}$ was assumed to be unity in the expansion of (25). If the expansion of the determinant does not contain the $n$th power of one of the variables, a linear transformation, independent of the coefficients of the form, will introduce such an $n$th power. The above proof then applies. The inverse of the linear transformation will restore the determinant to its original form without increasing the number of parameters.

Our knowledge concerning the particular sums $I_{\alpha \beta \gamma} \ldots$ which are independent gives immediately a limitation as to the forms which we may hope to express as determinants with linear elements. For $n=2$ or 3 , the coefficients $I_{\alpha \beta \gamma} \ldots$ are not all independent for $k>4$. For $n>3$ they are not all independent for $k>3$. Thus, we have

Theorem XI. Unless $k=2$ or $3, n$ any integer, or $k=4, n=2,3$, the general form is not expressible in determinantal form.

This is Dickson's second theorem.

Within the limitations just stated there is a one-to-one correspondence between the independent coefficients $I_{\alpha \beta \gamma} \ldots$ of the expanded form of (25) and the coefficients of the general form. Consequently, we have

THEOREM XII. In the field of all complex numbers the general binary form, the general ternary form, the general quaternary quadratic form, and the general quaternary cubic form can be expressed in determinantal form.

This is Dickson's fifth theorem, except that Dickson shows that the theorem is true for every binary form, every ternary form, and every quaternary quadratic form, regardless of the generality of the form.

The fact that the general quaternary cubic form can be expressed in determinantal form with linear elements leads at once to a theorem proved by H. Schröter* and also by Cremona;

THeOREM XIII. A sufficiently general cubic surface can be generated by three projective bundles of planes.

For the determinantal form of the cubic equation says at once that there exist three parameters $\lambda, \mu, \nu$ such that the three equations

$$
\lambda l_{i 1}+\mu l_{i 2}+\nu l_{i 3}=0 \quad(i=1,2,3)
$$

are satisfied by the coordinates of all points of the surface, where $l_{i j}$ are expressions linear in $x_{1}, x_{2}, x_{3}, x_{4}$.

* Journal für Mathematik, vol. 62 (1863), p. 265.

† Ibid., vol. 68 (1868), p. 79.

UNIVERSTTY OF KANSAS,

LawRence, Kansas. 